\title{
Data-oriented Media Service of Intelligent Car-to-x Communication in the Context of "Internet Plus"
}

\author{
Xu Zhiqiang ${ }^{1}$,He Jialiang ${ }^{2}$ and Wang Jiafu ${ }^{1}$ \\ ${ }^{1}$ Sichuan University of Media and Communications, China \\ ${ }^{2}$ Dalian Nationalities University, China
}

\begin{abstract}
The rapid development of Internet technology triggers a new-round revolution of automotive technology, while intelligent inter-connection will usher a new development trend of automotive technology. This paper analyses the current development of intelligent Car-to-x communication in the context of Internet Plus and the media effect of big data, and proposes that In-vehicle information system, as the fourth screen, may force auto electronics to transform from function type to information-service interacted type and eventually to evolve into an intelligent terminal with four wheels.
\end{abstract}

Keywords: Internet Plus, datamation, media service, intelligent Car-to-x communication, in vehicle information system

\section{Introduction}

In the past over one hundred-year history, revolution in automotive and traffic fields never stops. With the deepening of urbanization and the enhancing of people's living standard, people are spending increasing time on automotive's, thus higher requirement on vehicle entertainments are raised. Therefore intelligent Car-to-x communication is receiving more and more attention from people. The fourth screen, following TV set, computer and mobile phone will be the main application platform and a key development step of mobile Internet [1]. After mobile phone and PC being subverted by Internet, automotives will be the next target.

If the core competitiveness of traditional automotive industry is motor and tires, then the open, interactive and self-determined internet platform will lay a solid foundation for the humanization, software developing and intelligentization --the most important thing is the spirit of Internet products have already influenced consumers. Internet, or known as Car-to-x communication, is changing the living manners of vehicle owners, will redefine the interface, interactive means and board of mobile Internet, and even rebuild custom relationship (as shown in Figure 1). When we review the future automotives from the perspective of Internet, we will find it is no longer a simple transportation tool, but a super mobile intelligent terminal [2]. Year 2015 was the commencement of intelligent mobile which will enter a stage with high developing speed in the future, and relevant industry will reach an explosive growth. In September 2015, National Strategy Advisory Committee released Technical Route Map of Key Industries (Made in China 2025), in which the concept of "intelligent Car-to-x communication" was proposed from the level of national strategy ${ }^{1}$. On 2016 CES Exhibition, intelligent Car-to-x communication and relevant technologies were released, which aroused unprecedented attention from the market. In such an era, it is necessary to have a clearer idea of the current development of intelligent automotive in the context of Internet Plus. In particular, with the extension of full-touch in-vehicle operation system, wireless recharging and remote controlled parking

\footnotetext{
${ }^{1}$ Made in China 2025, Technical Route Map of Key Industries, National Strategy Advisory Committee released.
} 
technology, the intelligent service and data-oriented media service will bring more comfortable, convenient, relaxed and safer experience. Proceeding from the visual angle of Internet Plus Vehicle, based on analysis of intelligent Car-to-x communication development, this paper proposes that intelligent Car-to-x communication may offer a variety of data-oriented media services.

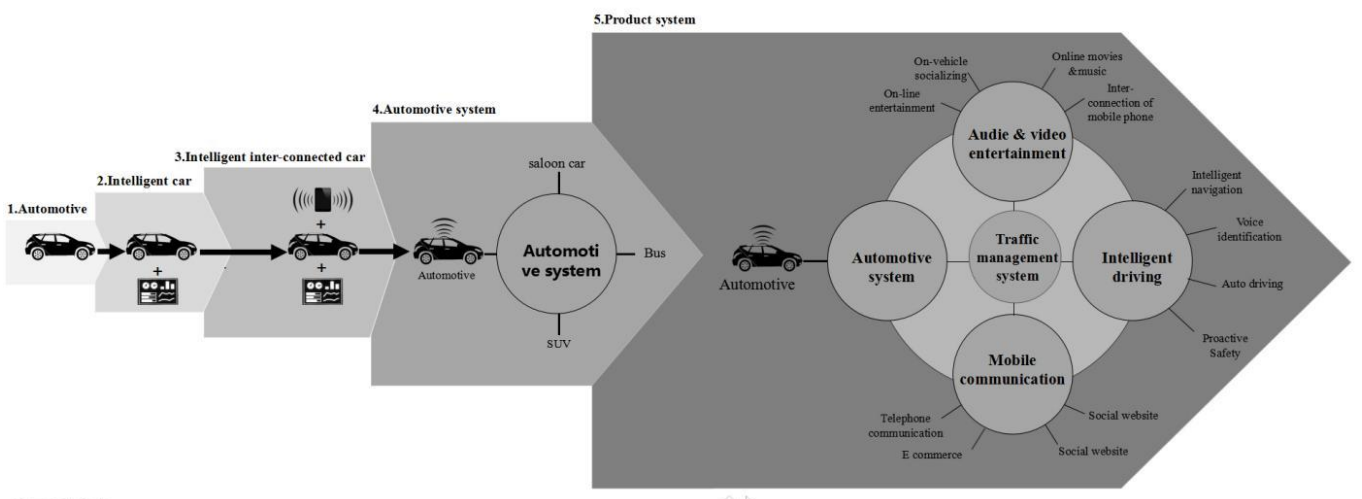

Figure 1. Car-to-x Communication Will Redefine Industrial Boundary

\section{Internet Plus and Intelligent Internet of Automotive}

\subsection{Internet Plus}

The birth of Internet drives information explosion and globalization. It is now returning back to Atom from Bit, fusing virtual and real word. Internet is also at an important turning presently. Premier Li Keqiang put forward the plan of "Internet Plus" ${ }^{2}$ for the first time in the National People's Congress and Chinese People's Political Consultative Conference this year, and commanded to drive the fusion of mobile Internet, cloud computation, big data, and Internet of Things with modern manufacturing industry.

Why Internet Plus traditional industry was escalated to a national strategic level? Internet plus market equals Taobao; Internet plus department store becomes Tmall; Internet plus taxi comes Didi, Kuaiche; and Internet plus traditional mate-making is Shijijiayuan. In addition to business industry, there are many possibilities in the future: Internet plus automotive, Internet plus transportation, Internet plus media and so on. "Plus" (+) is in fact a kind of capability, it is exterior resource and environment, the upgrade and transformation of traditional industry and cross-boundary fusion, with manman, man-object, man-service, man-scene, and man-future connected[3], forming a more extensive economic development state. By then the era of full digitalization will arrive[4] Thus Internet plus is the power source of traditional industry with the typical features of new infrastructure (cloud Internet), new production elements (data resource), new work division system (massive social synergism) etc. The effective combination of "cloud", "network" and "terminal" is an important approach driving new fundamental facility construction. "Cloud" refers to cloud computation and big data, particularly the safety assessment based on users' using habits, dynamic data and vehicle state; "network" includes Internet, Internet of things and Internet of service which connect man, things and service in a 3D network [5] (as shown in Figure 2) to actualize behavior matching and data gathering, with only data, low-cost sharing and transportation achieved, then utilized, commutated and assessed by cloud facilities. "Terminal" refers to the data source that can

\footnotetext{
${ }^{2}$ The so called Internet Plus: Put forward by Premier Li Keqiang in the third session of 12th National Congress, it is a new industrial pattern of Internet development under Innovation 2.0, Internet Evolvement driven by Knowledge Society Innovation, and a new social economic development form, and the fruits of further practices of Internet Concept.
} 
offer digitalized information and be identified by cloud system, at the same time is the physical interface or other forms serving users, including various mobile terminals and user-end APP. New infrastructure facility cloud terminal based on "Internet plus is showing in Figure 3.

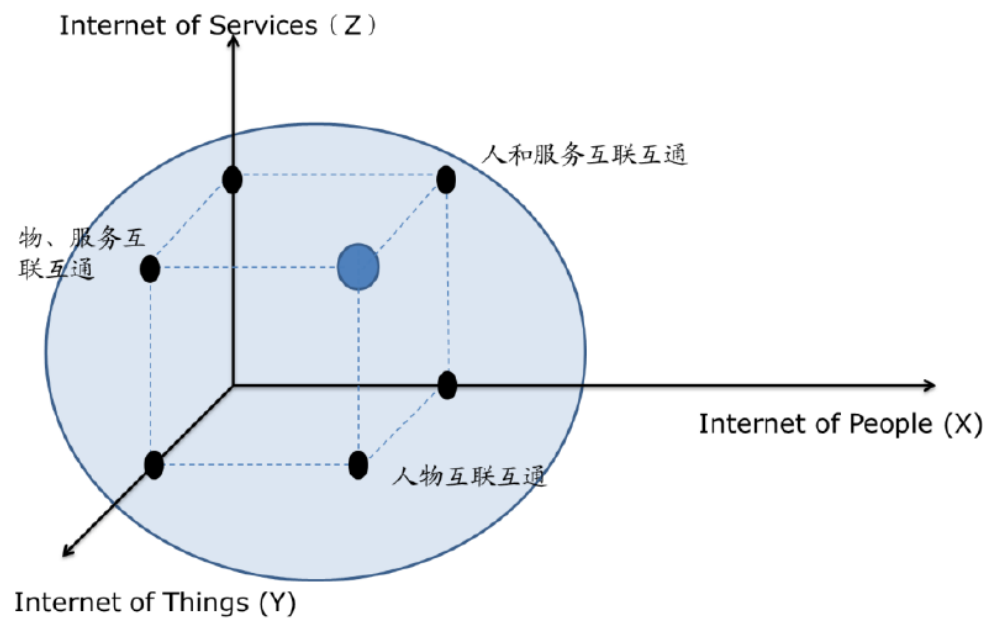

Figure 2. 3D Network of Internet of Human, Things and Services
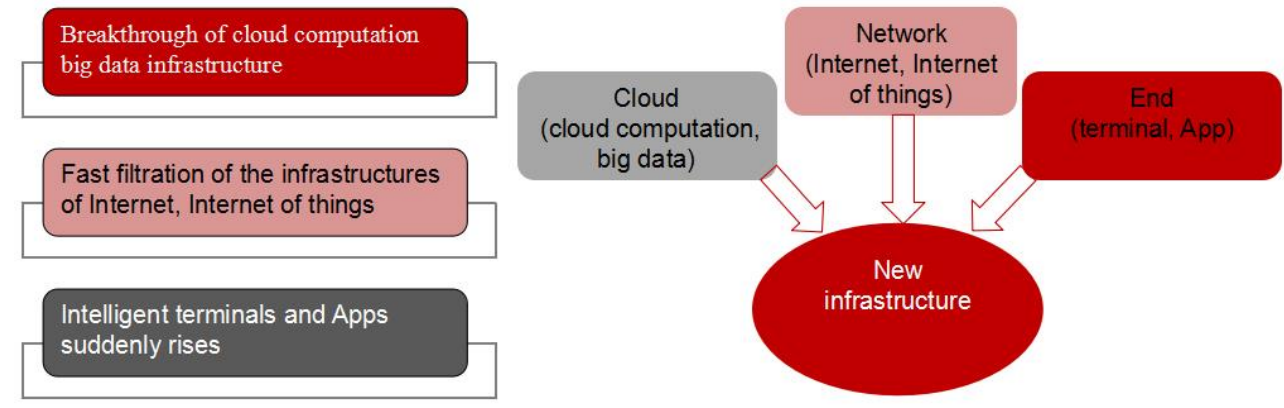

\section{Figure 3. New Infrastructure Facility Based on "Internet plus": Cloud Network}

\subsection{Intelligent Internet of Automotives}

Intelligent automotive is a comprehensive system integrating functions of environmental sensing, locating and navigating, route planning, sport controlling, planning decision making and multi-level driving assisting. [6] Since 2014, there are series of symbolic events in intelligent automotive industry. Google released its allfunction driverless car; Apple Inc. established a secrete R\&D center of Apple electric cars; Baidu proposed the solution to Car-to-x communication Carlife, and finished autodriving test on Road G7 in Beijing in December 2015; Volvo and Delphi completed highdegree auto driving tests; LeTV also announced to create a super car on which an economic framework integrating ecologic close-loop system (platform + content + terminal +application), cloud with multi screens, and inter-communication and cloud end to achieve seamless connection of smart devices such as automotives, mobile devices, $\mathrm{TV}$, and other devices can be worn by human[7].

The rapid development of Internet technology has triggered a new-round revolution of automotive technology. In the future, the correspondence technology will drive Internet of automotive to build automotives into a circulative ecologic system instead of the present closed state. Unprecedented changes will occur to automotive industry. On the way of seizing the fourth screen by Internet companies, in-vehicle information system mainly assumes the important tasks of vehicle-vehicle, vehicle-man and in-vehicle information 
processing, and is the core of intelligent automotive inter-connection and intelligentialize development. As a matter of fact, with the emerging of mobile Internet, mobile terminals and data sensors, data is growing at a high speed beyond our imagination [8]. According to the report by Advanced Television and the forecast by Juniper Research, by 2019 the mobile data generated by intelligent mobile phones and Tablets will be close to 197000 Gigabit, equal to the data volume of 10 trillion blu-ray movies.

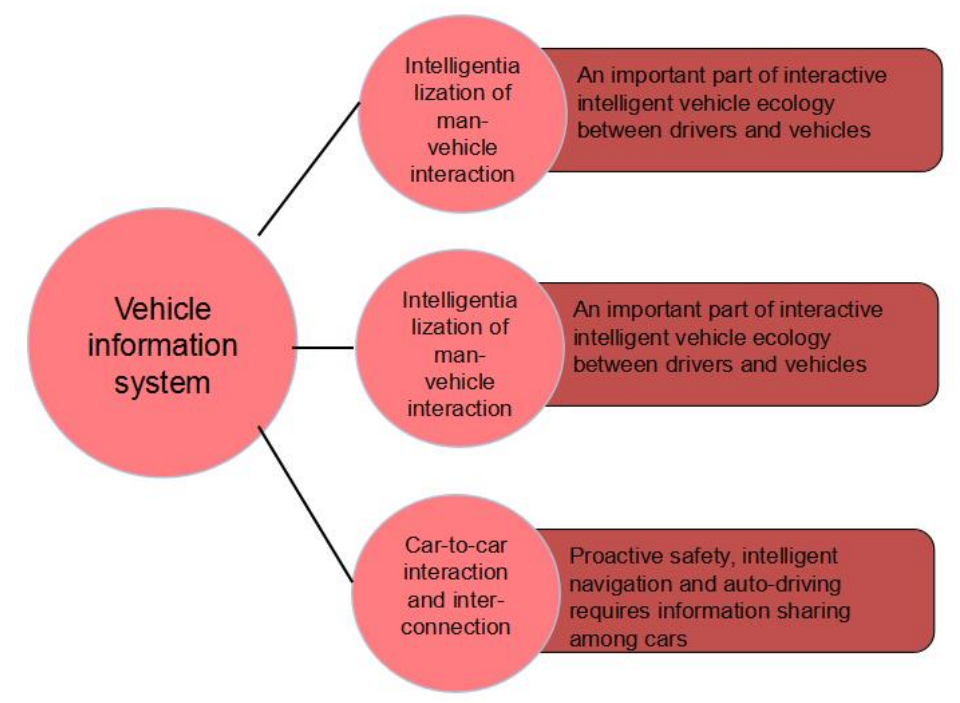

\section{Figure 4. In-vehicle Information System is the Core of Car-to-x Communication and Vehicle Intelligentialization}

\section{Data-oriented Medial Service of Intelligent Car-to-x Communication}

\subsection{Briefs of Big Data and its Media Effect}

In the development of big data, data are the source and driving power. Big data is changing our life, work and ways of thinking. Big data can be explained by four "Vs": Volume, Vast, Velocity and Variety [9]. In the meantime, the concepts of Veracity, Visualization and Value are supplemented here in this paper to better explain the wellprepared big data strategies. The first $\mathrm{V}$ emphasizes the huge data scale, and the second to the fourth $\mathrm{V}$ describe the vast endless space, different data types and fast data traffic and dynamic data system. The fifth to the seventh $\mathrm{V}$ tells the difficulty in judging the authenticity and accuracy of big data, the importance of visualization and the low value density. From data to knowledge, to value to synergistic mining to the final rapid decision making of value and service innovation, [10] Big data is so complicated and everchanging that the traditional data tools hard to capture, store, manage and analyze such data.

Fortunately artificial intelligence is capable of exponential computation, which is typically represented by the fact that the existing hardware, tools, algorithms can turn all raw data such as voice interaction, image detection (like face detection), mode detection and gesture detection into valuable data, with this the closed system will en gradually (as shown in Figure 5). With the effect of big data on media area, the "big data media" comes into being with the features of being supported by big data, enhanced knowledge-driven visualization, accurate orientation and data-oriented content etc. [11] 


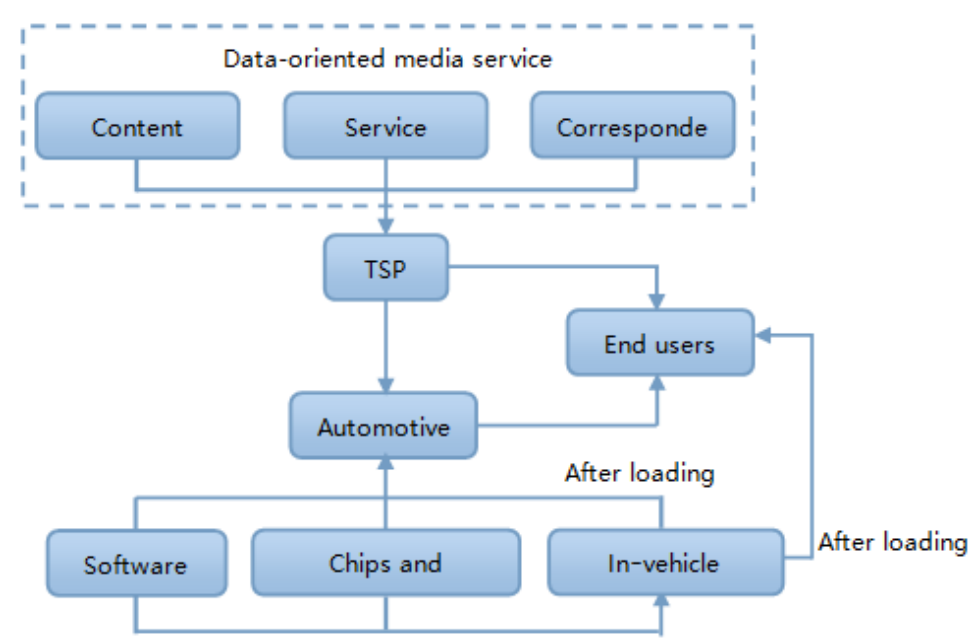

\section{Figure 5. Intelligent Internet Enables Closed Vehicle System to Open Gradually}

\subsection{Data-oriented Medial Service of Intelligent Connected Automotives}

Intelligent automotives, taking vehicle as the carrier, involves participation of crossboundary technologies like correspondence, software, information, analysis and detection etc. [12] In the future, automotives will be gradually evolved into information-service interactive electronic devices (with functions of audio and video entertainment, mobile correspondence, auto driving, life service and risk prevention) from functional devices (power control, vehicle control and safe driving control). In intelligent mobile robot [13] with functions of personal computers, Internet, cloud computation, Car-to-x communication, robot learning, and scene detection can be created and popularized in the next ten years. For example, the concept of industry $4.0^{3}$ undoubtedly presents a new visual angle of online manufacturing to automotive market. Drivers can not only actualize dialogs with vehicles through controller or voice input commands for air condition, top windows, entertainment, comfortableness and navigation control which can avoid risks arising from distraction, but also can control other functions of the vehicle through multitouch control and body gesture detection, including pinch zoom, touch screen action that can free drivers from driving completely and bring more convenient and safer driving experience.

Training of wireless Internet users' using habits by intelligent terminals, and the participation of Internet Companies like Apple Inc., Google Bat (Baidu, Alibaba and Tencent) will further accelerate the popularization and update of in-vehicle information system. In the future the industry boundary can be further expanded. By then Automotives is not only a data collector and sensor, but also the real-time information processor and releaser. Data of different dimensions are naturally inter-connected. Therefore, it is necessary to create a norm for map data collection, analysis and update. One important function of intelligent Car-to-x communication is sustainable and user-friendly vehicle-tovehicle $(\mathrm{V} 2 \mathrm{~V})$, vehicle-to-infrastructure (V2I), vehicle to person (V2P) and Vehicle-tosensor information interaction, then an informationalized ecological environment with multi-service and inner-connection among vehicles can be built, which will inevitably mark man-vehicle interaction-related media form with the feature of data-oriented. Intelligent car-to-X communication system will reconstruct the man-vehicle-road closedloop mode [14], thereby enhance the efficiency and safety of traffic system, which is a

\footnotetext{
${ }^{3}$ Industry 4.0: A Hi-tech Strategy Plan Proposed by Germany Government, means to utilize Cyber—Physical System to digitalize and intelligentialize the supply, manufacture and sales, and finally reach the goal of fast and effective supply of personalized products.
} 
revolutionary upgrade of automotive. Qualitative analysis of sensation, recognition, decision and implementation-based data-oriented media service is shown in Figure 6.

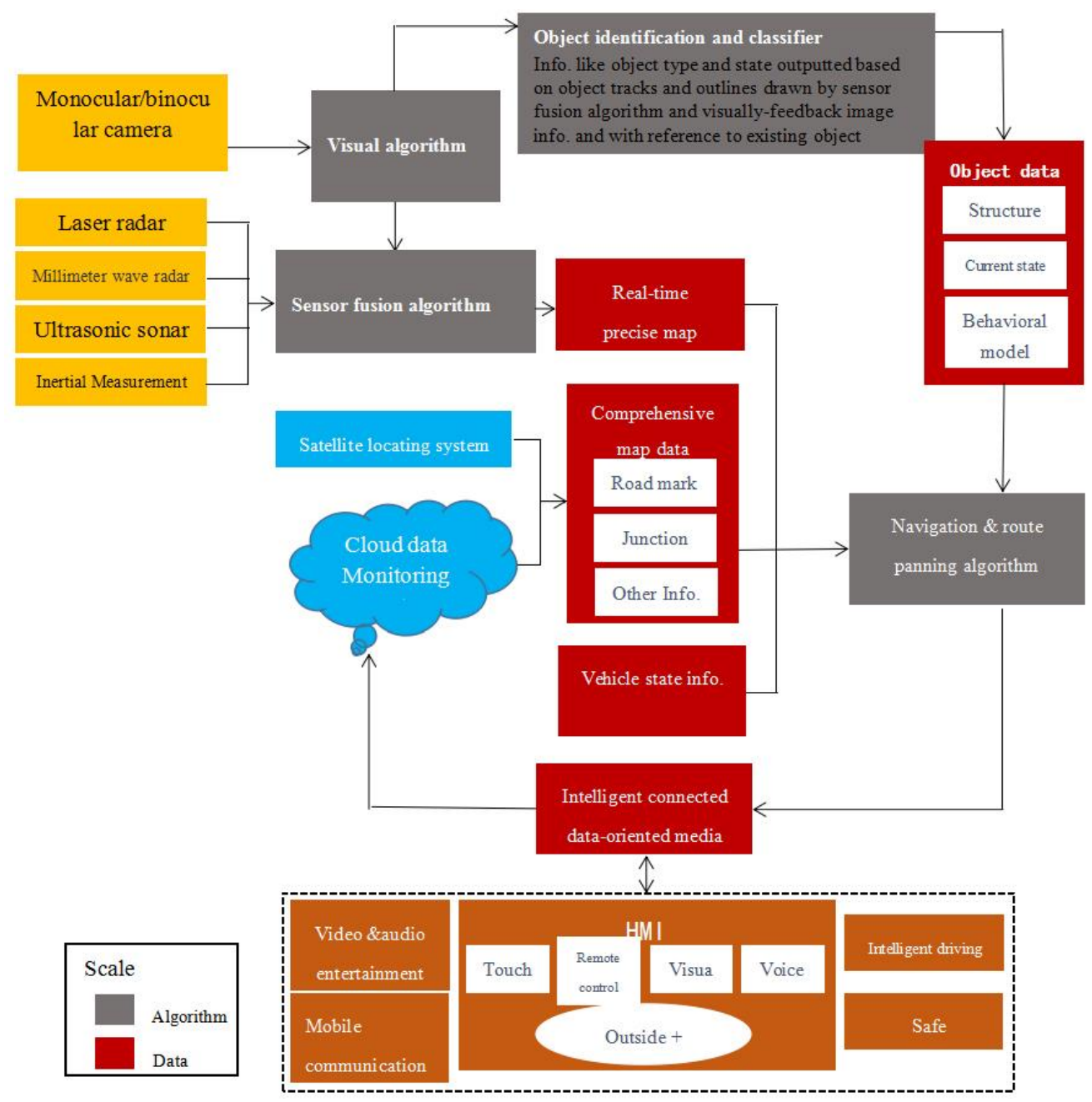

Figure 6. Qualitative Analysis of Intelligent Connected Automotive DataOriented Media Service

\subsubsection{Audio and Video Entertainment}

In recent years, in-vehicle information system is evolving into a multi-functional system integrating displaying, man-machine dialog and commanding \& control together from the traditional functions such as radio broadcasting, audio entertaining like MP3. Its main features are simultaneous processing of multiple multimedia, synchronization of processing and display, multiple screens and display. Soon a dual-screen in-vehicle information entertainment system may emerge; one screen actualizes functions of navigation, correspondence and setting, while the other is for in-vehicle music playing and temperature control.

The system can also receive all audio programs with Internet access. While using the system, uses can use voice command like saying "play (song title, singer, album or genre) to order the system to play specific songs they want, freeing us from classifying all these songs. For switching to another frequency of radio broadcasting, users can tell the name of radio station or the specific frequency; or users can turn on the volume by twisting, making a finger gesture in the air to pick up the phone etc. Thereby, the operation efficiency, convenience and safety of information entertainment system can be further 
enhanced. Besides, it can synchronize with the intelligent terminals in different scenes to share interested entertaining programs, daily schedules, individual itinerary, home and company locations, and so on.

Tomorrow automotives will be an entertaining and information service environment enabling video watching, music listening, game playing, shopping and stock investing etc.

\subsubsection{Mobile Correspondence}

Presently the information entertainment system has been the hub of the digital content, with wifi on which various information sources can be gathered. The latest 802.11acbased $5 \mathrm{G}^{4}$ WIFI and blue tooth can integrate users' mobile phones, wearable intelligent devices and players into the in-vehicle information terminal, and the capacitive touch screen will display the content shown on mobile devices and enables synchronized touch control. Vehicle owners can operate apps installed on their mobile phones through the capacitive touch screen, such as answering calls, reading and sending messages, playing music and video etc., eventually actualizing intelligent and convenient man-machine interaction experience and smooth HMI communication. These can be further explained in three levels: firstly adaptable assistance and inter-connection that depends on different driving environments; secondly data exchange based on vehicle-vehicle connection and man-machine interaction; thirdly the users' device, for example mobile phone or portable PC can be interconnected with automotives, including: Baidu navigation, music, Douban FM, umetrip, autohome etc. that can cover various demands like navigation, map, music listening, news, audio reading, voice interaction, traffic inquiry, maintenance and so on.

After connected with Iphone, seamless operation can be achieved through Siri EyesFree voice control. By pressing and holding a key for voice, drivers can turn to Siri for help, as simple as turning on siri while using iphone. This system is also equipped with function of automatically answering emergency calls, making the driving more convenient. At the same time, after the automotive enters users' own parking lane or garage, the system will download the latest upgrade package through wifi.

In the future, automotive will be a social network environment for correspondence, social networking, reading and sending emails, web page browsing or even QQ chatting.

\subsubsection{Intelligent Driving}

Car-to-x communication, as a specific application of Internet of things in transportation area, offers overall and comprehensive intelligent transportation services to users through multiple information fusion (RFID, GPS, Mobile communication, Wifi etc.). Sensor is similar to people's eyes, which is the foundation of driverless car and the carrier for sending environment. It can sense the objects around a car, such as other vehicles, pedestrians, red \& green lights on the road, and then feedback these info to the brain of automotives, achieving inter-connection of vehicles, men (driver and pedestrians), Car-to$\mathrm{x}$ communication platform and city network, accordingly bringing intelligent and safe driving and enabling users to enjoy technologies and life services etc. Moreover, highresolution map will actualize real-time big data fusion, verify the data received by sensors and assist the car to supervise the surroundings. In the future most cars will support autodriving. Transportation as a Service (briefed as TaaS) will be a new service model. City traffic jam will be improved $10 \%$ at least by then. [15] It is argued here that with the increasing demand of driverless cars, high-resolution online map will offer services in a cloud mode that can extract, gather and transmit huge real-data from various vehicle sensors.

\footnotetext{
${ }^{4} 5 \mathrm{G}$ is the key technology for achieving connection anywhere. $5 \mathrm{G}$ supports a volume of data up to $1000 \mathrm{~g}$, broadband speed per capita $10 \mathrm{Gbps}$, time lag no more than $1 \mathrm{~ms}$, all these features will flourish the development of new industry.
} 
According to McKinsey Global Institute, auto driving may bring a potential economic benefit amounting to 0.2-1.9 trillion USD, save 30-150 thousand persons' life from traffic accidents, and reduce annual carbon dioxide emission by 30 million ton. [16] Try to imagine, in the next 3-5 years, people's transportation means would be subverted completely: by clicking the mobile phone, the system will receive all road traffic information and dynamic navigation is enabled based on it; through 360-degree alldirection vision and the top 3D photographers, radar, laser and ultrasonic sensors, dynamic sensing of huge dynamic data can be acquired for real-time vehicle management of surroundings or even commanding the car for auto-drive to the users' place; on the way the vehicles can plan the route itself, and accelerate or decelerate speed automatically to avoid traffic jams or construction sites, or shift to other vehicle lanes, surpass or maintain a safe distance with other vehicles in order to reach the destination in the shortest time; after arriving the destination, by clicking the mobile phone, the vehicle will scan the parking lot and finish parking automatically; in the meantime the intelligent navigation system can display the real-time view of road entrances and 3D road sighs with voice reminding of road name. All these conduce to humane and convenient navigation experience. Therefore, high-resolution maps should be upgraded from time to time and downloaded to the vehicle system.

Soon vehicles will be an auto-driving environment capable of destination searching, importation time optimization, intelligent route panning, intelligent navigation and so on.

\subsubsection{Life Service}

Information sharing between vehicles and maintenance site will be actualized, which is also known as "inter-connected maintenance site": through in-vehicle terminal devices and cloud data center, the maintenance center can acquire real-time data of clients' vehicle heath state, including driving records, auto parts, system functioning state and so on. Besides, reminding of car maintenance can be sent to vehicle owners when necessary after logic algorithm of the driving records, past maintenance records and relevant professional technologies. The maintenance center has all necessary data of vehicles and relevant work staff can send information (such as maintenance records, route navigation and classic recommendation) requested by owners who can then have more understandings and information of the malfunctions of vehicles. Wireless recharging devices are installed in vehicles, users can recharge multiple devices once by placing the devices on the recharging panels. Furthermore, On-Board Diagnostic system can not only detect all physical data such as temperature, air flow, pressure, driving speed, driving courses, position, gas density, rotation rate and chassis state etc. [17], but also can describe the owners' habits, including pushing notifications on their heart rate, blood pressure, pulse, speed, and breath), all of which are of commercial value to some extent.

In the near future vehicles will be an overall life service environment capable of traffic supervision, traffic rule breaking record inquiry, in-vehicle payment, wifi connection, man service and location sharing and so on. 


\subsubsection{Safety Protection}

Internet of Things, cloud commutation, fast data connection and intelligent processing of big data brought new imagination space to the future market of automotive. Through the latest information technology, vehicle owners can experience safe driving (antisleeping driving system, anti-collision system, emergence alarm system), map navigation (online map, real-time road traffic, route rating, favorite items synchronizing, and realtime intercom), vehicle services (gas detection, vehicle inspection, driving course recording and maintenance reminding), intelligent setting (dynamic power, light air condition and door setting etc.), remote control and management (remote monitoring of vehicle location by APP, remote vehicle control, door locking/ unlocking etc.). In this way vehicle will be fused into intelligent life as a terminal of intelligent city and intelligent transportation system (ITS). Considering the fact the not all traffic situation can be informed in advance, virtual-real technology is needed to deal with all kinds of emergencies, such as frequent situations (based on analysis of millions of accidents) and low probability events that may bring disastrous consequence(like malfunction of sensors for auto driving and other irrational driving acts by drivers).

In the future automotive will be a safe environment of Internet of things that is capable of tracking, locating, driver monitoring, safety assessing, and transportation optimizing etc.

\section{Conclusions}

In the era of Internet plus, automotive industry will experience an unprecedented revolution. We believe in the near future, automotives can not only be driverless, but also achieve inter-connection with man, things, road and locations. Basically a dynamic balance and synergistic operation can be realized among men, vehicles and roads. In the city life of hustle and bustle, automotives will shuttle freely and orderly like fish under the direction of intelligent traffic, while drivers' feet, hands, eyes and even brains will be free from driving to handling business, watching movies or surfing the Internet. User experience will be improved further and traffic will be in order more than ever. All these are positive imaginations of future car-to- $X$ communication and data-oriented media service.

With the evolution and reform of automotive industry driven by car-to-X industry, data-oriented media service will bringing charming and best-than-ever user experience coupled with new challenges and opportunities. This will be the most exciting and expected exploring direction concerning the subject of humane, individualized and intelligent media service.

\section{Acknowledgments}

Key project of Sichuan Provincial Education Buerea Scientific Achievements Commercialization (16CZ0041); Chengdu science and technology project(No.2015HM01-00632-SF).

\section{References}

[1] Y. R. Lv, "Newspaper: Settled Down on Mobile Display Screen--note about Reading Newspaper on Electronic Devices", Journalists, vol. 12, (2010), pp. 68-72.

[2] L. D. Chen, "Driving Media Fusion by Internet", Contemporary Communication, vol. 6, (2014).

[3] Z. Q. Xu and H. D. Li, "Digital Media Fusion in the context of "Internet Plus and its Development Routes", China Press, no. 10, (2015), pp. 47-51.

[4] Negroponte, Editor, Y. Hu, translator, "Digitalized Survival”, Hainan Press, America, (1996).

[5] "The Federal Ministry of Education and Research, Recommendations for implementing the strategic initiative INDUSTRIE 4.0", (2013) April.

[6] D. Y. Chen, "Electrical Design and intelligent Technology for Modern Automotive", Modern 
Manufacturing Engineer, no. 2, (2008), pp. 134-137.

[7] "Super Automotive Comes! LeTV released intelligent automotive system" , http://auto.qq.com/a/20150121/011330.htm, (2015).

[8] X. Ding, "Big Data allows TV stations to understand Audiences better", Contemporary TV, no. 1, (2016).

[9] L. Peng, "ig Data Era: New Shaking faced by Journalism", Friends of Editor, no. 1, (2013).

[10] Z. Y. Pan, "7 Vs for Big Data Security-Basic Problem of Big Data and Information Security", China Information Security, no. 9, (2013).

[11] S. S. Cao, "Wearable data-oriented medial service in the context of media fusion", TV Technology, vol. 38, no. 22, (2014), pp. 21-23.

[12] Q. S. Chen, "A new Revolution of intelligent Product Consumption", Automotive Industry Report Shanghai Security, no. 12, (2014).

[13] R. X. Dou, “Triple play of intelligent Automotive Industry Pattern”, Internet Weekly, vol. 18, (2010), pp. 38-40.

[14] K. H. Guo, F. J. Ma and F. S. Kong, "Man-Vehicle-Road Closed-loop System Driver Model Parameters Identification", Automotive Engineering, vol. 1, (2002), pp. 20-24.

[15] "Global Connection Index 2025", Huawei, (2015).

[16] McKinsey Global Institute, "Disruptive technologies: Advances that will transform life, business, and the global economy", (2013) May.

[17] W. He, "Researches and Practices of Car-to-X Communication in the Era of Internet of Things", Science Press, (2013).

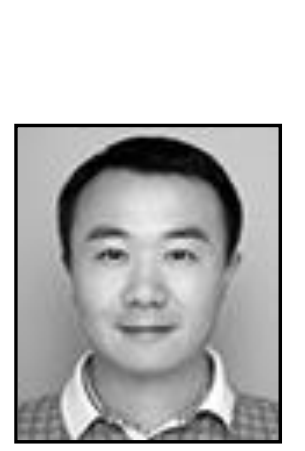

\begin{abstract}
Authors
Xu Zhiqiang, born in September 1981. He achieved his Master of Science degree of Technopreneurship \& Innovation Program (TIP) in Nanyang Technological University in Singapore. He also is an assistant professor of Sichuan University of Media and Communications, China. He is a Senior Member of Institute of Electrical and Electronics Engineers (IEEE) and experienced on both theorical studies and practices on the fields of mobile internet, all media business, micro-video and media big data, the internet of things and cloud computing integrated media business etc. Until now, $\mathrm{Xu}$ has published over 30 journals, authorized (applied) more than 30 national patents, and owns more than 30 copyrights of software.
\end{abstract}

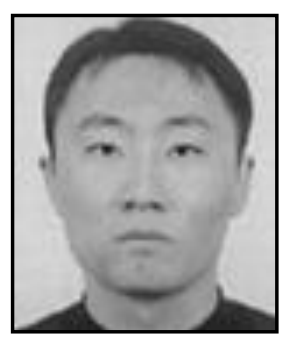

He Jialiang, was born in 1977 , received the $\mathrm{PhD}$ degree in computer software and theory from Jilin University of China in 2012 and the Master degree in computer application from Jilin University of China in 2004. Now he is an associate professor at College of Information and Communication Engineering, Dalian Nationalities University, China. His papers have been published in some wellknown international Journals and IEEE conferences. His main interests include Mobile Internet, Internet of Things, and Intelligent Business Information Processing.

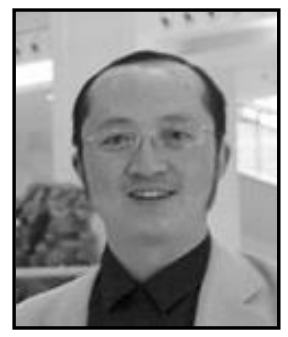

Wang Jiafu, Professor, master tutor, vice president, technical director, the executive director of the New Media Research Institute of Sichuan Institute of communications, digital media department. Specializing in 3D animation, interactive media, virtual reality, augmented reality, digital media and other related areas of research, teaching. 